\title{
GPS Satellite Clock Corrections without Relativity Theory
}

\author{
Stephan J. G. Gift \\ Department of Electrical and Computer Engineering, The University of the West Indies, St Augustine, \\ Trinidad and Tobago \\ Email: Stephan.Gift@sta.uwi.edu
}

How to cite this paper: Gift, S.J.G. (2021) GPS Satellite Clock Corrections without Relativity Theory. Journal of Applied Mathematics and Physics, 9, 2476-2482. https://doi.org/10.4236/jamp.2021.910158

Received: July 6, 2021

Accepted: October 15, 2021

Published: October 18, 2021

Copyright (c) 2021 by author(s) and Scientific Research Publishing Inc. This work is licensed under the Creative Commons Attribution International License (CC BY 4.0).

http://creativecommons.org/licenses/by/4.0/

\begin{abstract}
The GPS satellite clock corrections (along with gravitational redshift) which are necessary for the proper operation of the GPS are fully described without invoking relativity theory as is the practice today.

\section{Keywords}

GPS, Selleri Transformations, Time Dilation, Gravitational Time Dilation, Special Relativity, General Relativity
\end{abstract}

\section{Introduction}

In the Global Positioning System (GPS), there are atomic clocks on board the orbiting satellites which are necessary for the proper operation of the system. These clocks however experience clock retardation arising from their high-speed movement around the Earth (time dilation) and clock advancement resulting from reduced gravitational field intensity at the satellite altitudes (gravitational time dilation associated with gravitational redshift) [1]. These changes are today attributed to special relativity for the time dilation component and general relativity for the gravitational time dilation component. In order to compensate for the consequent time errors, the clock frequency standard on board each satellite must be appropriately offset at launch so that the clocks initially run slower than clocks on Earth [1] [2]. The IS-GPS-705F Interface Specification document [3] gives this adjusted frequency as $10.22999999543 \mathrm{MHz}$ down from $10.23 \mathrm{MHz}$. During operation, the effect of clock retardation and gravitational time dilation is an increase of the clock frequency from the offset value of 10.229999995543 $\mathrm{MHz}$ to $10.23 \mathrm{MHz}$ as seen by a ground observer. These corrections work extremely well and are extensively discussed in a Wikipedia entry titled "Error 
Analysis for the Global Positioning System" in the section "Relativity" [4]. This paper describes the clock corrections employed in the GPS along with gravitational redshift without invoking relativistic considerations as in [2] and [4] but using the data available there.

\section{Clock Retardation Due to Movement}

Based on accurate experimental data, it has been rigorously confirmed that clocks that are stationary in the GPS satellites run slow relative to clocks that are stationary in the ECI frame. For clocks at the same gravitational potential, this time reduction satisfies the equation [2]

$$
\int_{\text {path }} \mathrm{d} t^{\prime}=\int_{\text {path }}\left(1-\frac{v^{2}}{2 c^{2}}\right) \mathrm{d} t
$$

where $v$ is the velocity relative to the ECI frame, $t$ is the time in the ECI frame and $t^{\prime}$ is the time in the frame moving relative to the ECI frame which here is onboard the orbiting satellites. When integrated along the satellite path, assuming an approximately constant value for $v$, this equation gives

$$
t^{\prime}=\left(1-\frac{v^{2}}{2 c^{2}}\right) t
$$

This corresponds to the equation

$$
t^{\prime}=\frac{t}{\gamma}
$$

given by Kelly [5] where $\gamma=1 / \sqrt{1-v^{2} / c^{2}}$ since

$$
t^{\prime}=t / \gamma=t\left(1-\frac{v^{2}}{c^{2}}\right)^{1 / 2} \simeq t\left(1-\frac{v^{2}}{2 c^{2}}\right), v \ll c
$$

Equation (3) is the time transformation given by Selleri [6] [7] which is different from the Lorentz time transformation given by [8]

$$
t^{\prime}=\gamma\left(t-\frac{v x}{c^{2}}\right)=\frac{t}{\gamma}-\frac{v x^{\prime}}{c^{2}}
$$

where $x$ is the space coordinate in the ECI frame and $x^{\prime}$ is the space coordinate in the moving frame. Therefore, in order that satellite clocks remain synchronized with clocks that are stationary in the ECI frame (corresponding to the Earth's center), a continuous time adjustment has to be made to the satellite clocks. Thus from (3a), the difference between the time $t^{\prime}$ on the moving satellite clock and the time $t$ on the clock that is stationary in the ECI frame is given by

$$
t^{\prime}-t=-t \frac{v^{2}}{2 c^{2}}
$$

This represents a slowing of the clock rate as a result of the movement of the clock in the ECI frame. The velocity of the GPS satellites relative to the ECI frame is $v=3874 \mathrm{~m} / \mathrm{s}$. Therefore 


$$
t^{\prime}-t=t \frac{v^{2}}{2 c^{2}}=-t \frac{3874^{2}}{2\left(2.998 \times 10^{8}\right)^{2}} \simeq-8.349 \times 10^{-11} t
$$

Over a period of one day, the time change is given by

$$
t^{\prime}-t=-8.349 \times 10^{-11} \times 60 \times 60 \times 24 \simeq-7.214 \times 10^{-6} \mathrm{~s}=-7214 \mathrm{~ns}
$$

This means that the satellite clocks lose $7210 \mathrm{~ns}$ (rounded to $10 \mathrm{~ns}$ ) each day compared with the clocks that are stationary in the ECI frame as a result of movement at velocity $v=3874 \mathrm{~m} / \mathrm{s}$ relative to the ECI frame or the center of the Earth.

\section{Gravitational Redshift}

It has been experimentally observed that a light beam can change the momentum of an object upon which the light is incident. The corresponding pressure experienced by the object is referred to as radiation or light pressure. If the light energy $E_{p}$ is absorbed by the object, the momentum change experienced by the object is $E_{p} / c$. From the principle of conservation of momentum, this means that photons of energy $E_{p}$ behave as if they have a mass $m_{p}$ given by [9]

$$
m_{p}=\frac{E_{p}}{c^{2}}
$$

This derivation follows that given by Narlikar [10]. Consider a photon of mass $m_{p}$ leaving the surface of a massive object of mass $M$ and radius $R$ and escaping to infinity. At a distance $r$ from the center of the mass $M$, the force of attraction toward $M$ is

$$
F_{m_{p}}=\frac{G M m_{p}}{r^{2}}
$$

The work done in raising the photon through height $\mathrm{d} r$ is given by

$$
F_{m_{p}} \mathrm{~d} r=\frac{G M m_{p}}{r^{2}} \mathrm{~d} r
$$

Hence the work done in raising the photon from the surface of the massive object to an infinite height is given by

$$
W=\int_{R}^{\infty} F_{m_{p}} \mathrm{~d} r=\int_{R}^{\infty} \frac{G M m_{p}}{r^{2}} \mathrm{~d} r=\frac{G M m_{p}}{R}
$$

This work is done at the expense of the photon's energy. This loss of energy by the photon results in a reduction of its frequency from $f$ to $f^{\prime}$ given by

$$
h f-h f^{\prime}=\frac{G M m_{p}}{R}
$$

Using (8), this becomes

$$
h f-h f^{\prime}=\frac{G M h f}{R c^{2}}
$$

where $E_{p}=h f$. Hence the fall in frequency $\Delta f=f-f^{\prime}$ is given by 


$$
\Delta f=\frac{G M}{R c^{2}} f
$$

This frequency change is referred to as gravitational redshift and has been confirmed to an accuracy of $1 \%$ or better [11].

\section{Clock Advancement Due to Reduced Gravity}

The work done in raising the photon from an initial height $r=R_{1}$ to an increased height $r=R_{2}$ is given by

$$
W=\int_{R_{1}}^{R_{2}} F_{m_{p}} \mathrm{~d} r=\int_{R_{1}}^{R_{2}} \frac{G M m_{p}}{r^{2}} \mathrm{~d} r=-\frac{G M m_{p}}{R_{2}}+\frac{G M m_{p}}{R_{1}}
$$

Therefore, the fall in frequency is given by

$$
\Delta f=f-f^{\prime}=\frac{G M f}{c^{2}}\left(\frac{1}{R_{1}}-\frac{1}{R_{2}}\right)
$$

Now if a radiation source emits photons at frequency $f$ over time $t$ registered on a clock at $R_{1}$, then the number of cycles emitted is $N=t f$. If these photons travel to a greater height, then the frequency falls to $f^{\prime}$. However, since the receiver must receive the same number of cycles, it follows that [12]

$$
t f=N=t^{\prime} f^{\prime}
$$

where $t^{\prime}$ is the time registered on a clock at $R_{2}$. Therefore, the decrease in light frequency is associated with an increase in clock rate at that position. This change in clock rate is referred to as gravitational time dilation. From (16) and (17),

$$
t^{\prime}=\frac{t f}{f^{\prime}}=t \frac{f}{f-\frac{G M f}{c^{2}}\left(\frac{1}{R_{1}}-\frac{1}{R_{2}}\right)} \simeq t\left[1+\frac{G M}{c^{2}}\left(\frac{1}{R_{1}}-\frac{1}{R_{2}}\right)\right], \frac{G M}{c^{2} R} \ll 1
$$

Equation (18) has been confirmed by accurate GPS data [2]. Specifically, for $M=M_{\text {Earth }}, R_{1}=R_{\text {Earth }}$ and $R_{2}=R_{\text {Sat }}$, the clocks on the satellites in the GPS run faster as a result of the decreased gravitational field intensity according to Equation (18).

Therefore, in order that satellite clocks remain synchronized with clocks at rest on the surface of the Earth, a continuous time adjustment has to be made to these clocks. Thus from (18),

$$
t^{\prime}=t \frac{1}{1-\frac{G M_{\text {Earth }}}{c^{2}}\left(\frac{1}{R_{\text {Earth }}}-\frac{1}{R_{\text {Sat }}}\right)} \simeq t\left[1+\frac{G M_{\text {Earth }}}{c^{2}}\left(\frac{1}{R_{\text {Earth }}}-\frac{1}{R_{\text {Sat }}}\right)\right], \frac{G M}{c^{2} R} \ll 1
$$

Hence the difference between the time $t^{\prime}$ on the moving satellite clock and the time $t$ on the clock on the surface of the Earth is given by

$$
t^{\prime}-t=t \frac{G M_{\text {Earth }}}{c^{2}}\left(\frac{1}{R_{\text {Earth }}}-\frac{1}{R_{\text {Sat }}}\right)
$$


Therefore, with mass of the Earth $M_{\text {Earth }}=5.974 \times 10^{24} \mathrm{~kg}$, polar radius of Earth $R_{\text {Earth }}=6.357 \times 10^{6} \mathrm{~m}$, radius of satellite orbit $R_{\text {Sat }}=2.6541 \times 10^{7} \mathrm{~m}$, $G=6.674 \times 10^{-11} \mathrm{~m}^{3} \cdot \mathrm{kg}^{-1} \cdot \mathrm{s}^{-2}$ and $c=2.998 \times 10^{8} \mathrm{~m} / \mathrm{s}$ we get

$$
\begin{aligned}
t^{\prime}-t & =t \frac{G M_{\text {Earth }}}{c^{2}}\left(\frac{1}{R_{\text {Earth }}}-\frac{1}{R_{\text {Sat }}}\right) \\
& =t \frac{6.674 \times 10^{-11} \times 5.974 \times 10^{24}}{\left(2.998 \times 10^{8}\right)^{2}}\left(\frac{1}{6.357 \times 10^{6}}-\frac{1}{2.6541 \times 10^{7}}\right) \\
& \simeq 5.307 \times 10^{-10} t
\end{aligned}
$$

Over a period of one day, the time increase is given by

$$
t^{\prime}-t=5.307 \times 10^{-10} \times 60 \times 60 \times 24 \simeq 45.850 \times 10^{-6} \mathrm{~s}=45850 \mathrm{~ns}
$$

This means that the satellite clocks gain 45,850 ns each day compared with the clocks that are stationary on the surface of the Earth as a result of the decreased gravitational field intensity above the Earth [2].

\section{Clock Frequency Adjustment}

The net time gain each day from clock movement and reduced gravitational field intensity is then $45850-7210=38640 \mathrm{~ns}$. The satellite clocks must be appropriately slowed in order to exactly compensate for this net time gain. From Equations (5) and (20), the total time change resulting from clock movement and gravitational time dilation is given by

$$
t^{\prime}-t=t \frac{G M_{\text {Earth }}}{c^{2}}\left(\frac{1}{R_{\text {Earth }}}-\frac{1}{R_{\text {Sat }}}\right)-t \frac{v^{2}}{2 c^{2}}
$$

Therefore, the fractional change in time is given by

$$
\begin{aligned}
\frac{t^{\prime}-t}{t} & =\frac{G M_{\text {Earth }}}{c^{2}}\left(\frac{1}{R_{\text {Earth }}}-\frac{1}{R_{\text {Sat }}}\right)-\frac{v^{2}}{2 c^{2}} \\
& =5.307 \times 10^{-10}-8.349 \times 10^{-11} \\
& =4.472 \times 10^{-10}
\end{aligned}
$$

In order to correct for this, the clock frequency must be reduced by the same fraction i.e.

$$
\frac{f-f^{\prime}}{f}=\frac{t^{\prime}-t}{t}=4.472 \times 10^{-10}
$$

where $f^{\prime}$ is the reduced frequency and $f=10.23 \mathrm{MHz}$ is the clock frequency on Earth. From (25) therefore,

$$
\begin{aligned}
f^{\prime} & =f\left(1-4.472 \times 10^{-10}\right) \\
& =10.23 \mathrm{MHz} \times\left(1-4.472 \times 10^{-10}\right) \\
& =10.22999999543 \mathrm{MHz}
\end{aligned}
$$

This is the adjusted frequency that is preset in the clocks at launch and can be found in IS-GPS-705F Interface Specification, May 2019 (page 9) [3]. Section 3.3.1.1 states "The carrier frequencies for the L1, L2 and L5 signals shall be co- 
herently derived from a common frequency source within the SV [space vehicle]. The nominal frequency of this source-as it appears to an observer on the ground-is 10.23 MHz. The SV carrier frequency and clock rates-as they would appear to an observer located in the SV-are offset to compensate for relativistic effects. The clock rates are offset by $\Delta f f=-4.4647 \mathrm{E}-10$, equivalent to a change in the I5 and Q5-code chipping rate of $10.23 \mathrm{MHz}$ offset by a $\Delta f=-4.5674 \mathrm{E}-3$ $\mathrm{Hz}$. This is equal to $10.2299999954326 \mathrm{MHz}$. Thus, the effect of the clock retardation and gravitational time dilation is to cause the clock frequency to increase from the offset value of $10.22999999543 \mathrm{MHz}$ to $10.23 \mathrm{MHz}$ as seen by an observer on the ground.

\section{Conclusion}

In this paper, the clock corrections necessary in the GPS have been calculated without using relativity theory. As previously indicated, the speed of the satellite clocks is measured relative to the ECI frame corresponding to the center of the Earth or the poles. It turns out that (because of time change resulting from gravity and clock movement) clocks at the poles beat at the same rate as clocks along the Earth's geoid across the surface of the Earth. This means that clocks that are stationary in the ECI frame are equivalent to clocks being stationary anywhere on the Earth's geoid, regardless of latitude [2]. This is a very convenient result!

\section{Conflicts of Interest}

The author declares no conflicts of interest regarding the publication of this paper.

\section{References}

[1] Kaplan, E. and Hegarty, C. (2017) Understanding GPS/GNSS: Principles and Applications. $3^{\text {rd }}$ Edition, Artech House Publishers, London.

[2] Ashby, N. (2003) Relativity in the Global Positioning System. Living Reviews in Relativity, 6, Article No. 1. https://doi.org/10.12942/lrr-2003-1

[3] IS-GPS-705F Interface Specification (May 2019). https://navcen.uscg.gov/pdf/gps/IS_GPS_705F.pdf

[4] Wikipedia (2021) "Error Analysis for the Global Positioning System" in the Section "Relativity". https://en.wikipedia.org/wiki/Error_analysis_for_the_Global_Positioning_System

[5] Kelly, A. (2005) Challenging Modern Physics. Brown Walker Press, Florida.

[6] Selleri, F. (1997) Noninvariant One-Way Speed of Light and Locally Equivalent Reference Frames. Foundations of Physics Letters, 10, 73-83. https://doi.org/10.1007/BF02764121

[7] Gift, S.J.G. (2015) The Selleri Transformations and the One-Way Speed of Light. Physics Essays, 28, 474-481. https://doi.org/10.4006/0836-1398-28.4.474

[8] Rindler, W. (2006) Relativity Special, General and Cosmological. $2^{\text {nd }}$ Edition, Oxford University Press, New York.

[9] Skinner, R. (1982) Relativity for Scientists and Engineers. Dover, New York. 
[10] Narlikar, J.V. (1982) Violent Phenomena in the Universe. Oxford University Press, Oxford.

[11] Pound, R. and Rebka, G. (1960) Apparent Weight of Photons. Physical Review Letters, 4, 337. https://doi.org/10.1103/PhysRevLett.4.337

[12] Sartori, L. (1996) Understanding Relativity. University of California Press, California. 